\title{
Evaluación de la investigación en ciencias sociales Una trayectoria y dos caminos posibles
}

\author{
Teresa Pacheco-Méndez ${ }^{1}$ \\ kat_tpm@yahoo.es \\ Rec. 15/11/2017, Apr. 22/03/2018
}

\section{Introducción}

La práctica de la evaluación es y se ha convertido en un tema muy debatido en diversos sectores de la sociedad. Desde el interés depositado en ella para incrementar la productividad y competitividad propias del mundo empresarial, hasta su papel jugado como componente determinante en la definición del rumbo de la ciencia y la cultura, la evaluación se ha desempeñado como una herramienta en extremo versátil, amoldándose a cuanta variedad de espacios institucionales han requerido de ella para el logro de fines particulares. En este trabajo se abordan dos perspectivas de la evaluación que afectan directamente a la investigación universitaria en ciencias sociales: la perspectiva de la administración, y otra que apunta a las posibilidades reflexivas de la evaluación. Con tal propósito se exponen algunas puntualizaciones con respecto al tema de los juicios de valor, sobre el objeto-objetivo-utilidad de la evaluación, y por último sobre sus posibilidades innovadoras en el terreno del conocimiento.

En la perspectiva de la organización administrativa, la evaluación se encuentra estrechamente ligada a prácticas de Estado y a términos tales como información y control. Para los expertos en este campo, "los datos son las representaciones más directas sobre los hechos, mientras que las informaciones propiamente dichas son aquellas en las que los datos han sido interpretados o elaborados de alguna manera" (Hintze, 2001, pp. 2930). Por su parte, el control, además de operar con información, tiene la encomienda de "verificar los hechos (mediante el registro de tales informaciones), de manera que sea posible compararlos con algún patrón técnico de referencia; esto proporciona un nuevo tipo de información resultado de la comparación entre patrones técnicos y datos de la realidad" (Hintze, 2001, pp. 30-31). Si bien la evaluación contempla el control, ésta también es portadora de juicios de valor implícitos y explícitos, "Si controlar es comparar informaciones de la realidad contra patrones de referencia técnicos, evaluar será comparar tales informaciones contra patrones de referencia valorativos" (Hintze, 2001,

\footnotetext{
1 Socióloga con Doctorado en Pedagogía (Mención honorífica). Desde 1988 es Miembro del Sistema Nacional de Investigadores (actualmente cuenta con el Nivel II). Es Investigadora Titular " $C$ " de tiempo completo en el Centro de Estudios Sobre la Universidad de la UNAM con una antiguedad de 26 años en tareas de investigación. Ha sido profesora de las Divisiones de Estudios de Posgrado de las Facultades de Filosofía y Letras y de Ciencias Políticas y Sociales, ambas de la UNAM.
} 
p. 31). Es precisamente esta facultad de juzgar, la que da lugar a efectos cruciales de los procesos de evaluación emprendidos; así, en el caso de la evaluación de la investigación social (Birman, 2010), el pensamiento y el entendimiento se enfrentan al riesgo de ser reducidos a la razón instrumental.

La evaluación, entendida como dispositivo para la gestión de recursos, se desempeńa como un operador de legitimidad fundado en un imaginario normativo que le da sentido, confiriéndole a los actores y a los espacios institucionales que lo contienen, su justificación social (Eraly, 2016). De ahí que toda reflexión sobre la evaluación -visualizada desde la perspectiva del control- haya encontrado su principal plataforma en el reconocimiento de tres de sus componentes: un proceso orientado a delimitar y obtener informaciones sobre el valor de una acción (conocer); un producto que es el juicio de valor (juzgar) y, por último, el uso de tal producto para modificar la acción a través de decisiones (decidir) (Shadish 1998, en Demarteau, 2007, p. 27).

En tiempos más recientes (principalmente durante las décadas 1960, 1970 y 1980), la evaluación se consolida como una práctica sistemática y objetiva, principalmente en los dominios de la educación y la salud. Su institucionalización y profesionalización a gran escala, abre espacios formales a sociedades prestadoras de servicio, redes profesionales y formaciones especializadas, como así también al diseño de modelos de evaluación y al establecimiento de centros de estudio. (Demarteau, 2007). Muy pronto, la evaluación encuentra un nuevo respaldo tanto en la comparación entre diferentes modelos, como en prácticas más participativas por parte de los actores intervinientes.

Una distinción más entre evaluación y control es la establecida por Ardoino y Berger (1998), sostienen que:

El objetivo de los procedimientos de control es el de comparar el grado de conformidad, si no es de identidad, entre un modelo de referencia y los fenómenos que vencen, u ocurrentes, como testifica claramente la etimología contable de la palabra: la contra verificación, el registro doble que permite verificar la contabilidad. [...] El control se centra en la coherencia y en la homogeneidad. $(\mathrm{s} / \mathrm{p})$

Por el contrario, la evaluación se ubica en términos de significancia y significado. Mientras el control reposa en dispositivos construidos, y lo más transparentes posible, la evaluación se representa como un processus y como un trámite que compromete siempre y necesariamente, zonas irreductibles de opacidad (Ardoino y Berger, 1998).

La prevalencia de las iniciativas institucionales de control estatal sobre las diversas actividades sociales y la evaluación de sus resultados, tiende a hacerse más presente cuando el propósito es afianzar y legitimar los modos de producción de la modernidad actual. "La evaluación es un modo a través del cual un poder (político o administrativo, general o local) ejerce su imperio sobre el saber y el saber hacer, factores que presiden a las diferentes actividades pretendiendo administrar la norma de lo verdadero" (Zarka, 2009, p. 4). La influencia de este proceso en las ciencias sociales influye de manera decisiva en las perspectivas de desarrollo de este ámbito de conocimiento. Habermas lo expresa de la siguiente manera "[...] cuanto más vienen determinados el crecimiento y el cambio de la sociedad por la racionalidad externa propia de los procesos de investigación desarrollados en un régimen de división de trabajo, tanto menos se asienta la civilización científica sobre el saber y la conciencia de sus ciudadanos" (Habermas, 1987, p. 220). 
Lo que hasta la fecha ha quedado puesto a la luz -aunque no con la suficiente convicción y consenso- es que la evaluación descansa tanto en una inter-subjetividad de puntos de vista, como en una considerable cantidad de datos provenientes de usuarios, destinatarios, profesionales, y de otros sectores de la sociedad que en ella intervienen. En este cruce de inter-subjetividades inevitablemente se expresan, se exponen y se ponen en juego los intereses de unos y de otros: sus respectivas divergencias, convergencias, complementariedades y contradicciones; un escenario que invita a pensar en una visión distinta de la evaluación que contemple sus dimensiones epistemológicas y sociohistóricas.

\section{El componente epistemológico y sociohistórico}

Un punto de partida para reflexionar sobre el sentido y alcance de la evaluación consiste, precisamente, en esclarecer sus respectivos fundamentos como mecanismo de control y como proceso. Ardoino y Berger (1998) proponen una distinción sistemática entre los processus de evaluación y los procedimientos de control, estableciendo como un elemento de referencia las variables espacio/tiempo. Para los autores, el control se centra en el espacio y fuera del tiempo, condición indispensable como base de medida que permite su factorización. En cambio, la evaluación se caracteriza por su processus mismo, que es indisociable de la vivencia histórica y temporal. En este último sentido, Birman (2010) coincide en que los registros del tiempo y del símbolo están íntimamente conjugados en la constitución del sujeto, de tal suerte que estos últimos configuran al símbolo en su dimensión histórica.

Otro rasgo que contribuye a replantear el sentido de la evaluación -en franca discrepancia con el control- es el esclarecimiento de su referente de realidad con respecto a la acción del sujeto y a los juicios de valor.

A) La "evaluación/control" descansa en la identificación y selección de espacios segmentados de la realidad objetivada, traducidos a conceptos de referente cerrado, fincados en acciones no diferenciadas realizadas por los sujetos -todo ello con fines de medición, verificación y sistematización-. En este caso, la intersubjetividad y la libertad como cualidad fundamental del sujeto y del ciudadano, quedan al margen de toda consideración. Esta cualidad lo convierte en un instrumento de "evaluación/control" hoy día idóneo para la resolución de problemas y exigencias concretas del sector público en sus distintos ámbitos institucionales de educación, salud, vivienda, deporte, economía, $\mathrm{y}$ de gobierno en general.

La evaluación como proceso se plantea más bien como una estrategia de conocimiento de lo social aparejada a su dinámica de movimiento y transformación. Su propósito, más allá de trascender las fronteras institucionales y administrativas, se sitúa en la singularidad del sujeto, un rasgo que encierra diferencias y contrastes interpersonales, de grupo, y de comunidades - muy al contrario de la tendencia homogeneizadora de la "evaluación/control", donde el sujeto queda privado de la libertad de ejercicio de su pensamiento- (Birman, 2010). La evaluación como proceso se apoya en la indisociable experiencia histórica y de tiempo que da contexto y sentido a toda actividad humana.

B) La evaluación contempla la posibilidad para diferenciar juicios de valor. Al respecto, Leclerc (2016) se suma a distinción establecida por Kant sobre los juicios determinantes y los reflexivos. Mientras los juicios determinantes o de conocimiento se distinguen por aplicar a los datos empíricos nuevos conceptos y categorías teóricas pre- 
viamente disponibles, el juicio estético es un juicio reflexivo que no se limita a aplicar conceptos construidos, sino que también se interroga sobre la singularidad de lo que debe juzgar, así como de los conceptos que deberían guiar su juicio.

Tal distinción epistemológica sigue hoy día presente, encontrando su fundamento en el desarrollo actual de los modernos sistemas de producción. La modernidad como experiencia histórica de la sociedad es caracterizada por Giddens como "un orden postradicional en el que, [...] la seguridad de tradiciones y costumbres no ha sido sustituida por la certidumbre del conocimiento racional" (Giddens, 2000, p. 11). Presenciamos una modernidad donde el cambio social avanza a mayor velocidad que en sistemas anteriores, cimentando y asegurando su dinámica a través de la interacción de tres elementos: la separación entre tiempo y espacio, el desenclave respaldado por los sistemas abstractos y, por último, la reflexividad institucional. En palabras del autor: "La transformación de tiempo y espacio, unida a los mecanismos de desenclave, liberan la vida social de la dependencia de los preceptos y prácticas establecidas" (Giddens, 2000, p. 33).

Por desanclaje de los sistemas y las instituciones sociales, Giddens (1994) explica que se trata de un "despegar" las relaciones sociales de sus contextos locales de interacción para entonces reestructurarlas en indefinidos intervalos espacio-temporales. Para él, los mecanismos de desenclave son los denominados sistemas expertos cuya función es disociar la interacción social de las peculiaridades de lo local; en este caso se encuentran los programas de evaluación a gran escala, las evaluaciones de sistemas de servicios y políticas públicos, y la evaluación institucional, principalmente.

Giddens expone que el desafío al que se enfrenta la sociedad ante la presencia e incidencia de los sistemas abstractos consiste precisamente en aprender a manejar este y otros riesgos de la sociedad moderna. Este esfuerzo se hace posible a partir de la "reflexividad" del saber social, una tarea donde las prácticas sociales son examinadas constantemente, y reformadas a la luz de nueva información sobre ellas mismas, alterando de esta manera, su carácter constituyente. La "[...] reflexión de la modernidad, de hecho, derriba la razón, siempre que se entienda por razón la obtención de un conocimiento cierto" (Giddens, 1994, p. 47).

Este "[...] experimento global de la modernidad influye en y es influido por la penetración de las instituciones modernas en el tejido de la vida cotidiana. No es solo la comunidad local, sino también los rasgos íntimos de la vida personal y del yo los que se entretejen con relaciones de extensión espacio-temporal indefinida" (Giddens, 2001. p. 79). La reflexividad de la modernidad alcanza al corazón del yo... Las transiciones en las vidas individuales han exigido siempre una reorganización psíquica, algo que en las culturas tradicionales solía quedar ritualizado en forma de ritos de paso (Giddens, 2000).

Tanto la investigación social como la evaluación actúan sobre la trayectoria histórico-institucional de los sujetos, y tan sólo de manera muy indirecta ambas actividades cuentan con la posibilidad de reorientar por sí mismas sus objetivos, en la perspectiva de formular escenarios futuros de carácter instituyente; es aquí donde radica la capacidad y fuerza de la acción de los individuos, así como el potencial de la evaluación como plataforma para la promoción de la investigación en ciencias sociales. 


\section{La evaluación como proceso}

Hasta este punto, hemos identificado dos sentidos y propósitos de la evaluación: por un lado, situándola en la perspectiva de la realidad y de la acción social e institucional como algo dado -establecido, predecible, controlable, exterior y ajeno a la voluntad de los individuos-; y por otro, posicionándola en una realidad donde lo dado es trascendido por una dinámica activada por la acción social de los individuos. Aun cuando ambas son concepciones epistemológicas respaldadas por referentes de realidad muy distintos -debido a la función de los paradigmas sobre los cuales cada una de ellas descansa-, las dos funcionan de manera entrelazada y son prácticamente indisociables, interdependientes e idealmente complementarias.

Si el propósito de la evaluación de la investigación en ciencias sociales es situarla en el orden del "dándose" histórico, el sentido y las significaciones de coherencia y compatibilidad estarán estrechamente asociados con los modelos científicos, institucionales, culturales y sociales prevalecientes en este campo de actividad. El sistema o los sistemas de referencia implicados en los fenómenos a evaluar deben ser, por lo tanto, reelaborados y reconstruidos como sistemas de referencia dinámicos y diferenciados, pero también orgánicamente articulados con la experiencia de los individuos.

Tales sistemas de referencia constitutivos del proceso de evaluación no son exteriores (espacial y lógicamente hablando) ni anteriores (cronológicamente hablando) a los sujetos y a los fenómenos como lo concibe la lógica del control. Por el contrario, la evaluación como proceso multi-referenciado se regula por la contingencia histórica, la heterogeneidad de sus componentes, y la intersubjetividad constituyente. Toda significación proveniente de los procesos involucrados en la evaluación requiere de la construcción de una red de relaciones de órdenes sociales diversos, organizacionales, comunitarios, de grupo e interpersonales. De ahí que el diseño del procedimiento y la selección de los instrumentos de análisis, de los indicadores, así como de los agentes responsables de la evaluación, varíe considerablemente en función tanto de la naturaleza de los objetos por evaluar, así como de sus respectivos sistemas de referencia (cognitivos, históricos, políticos, culturales institucionales, de grupo y de relación interpersonal).

Evaluar algo o a alguien significa elaborar y formular una estimación o una apreciación al respecto. Para Friedberg (1993, en Maury et. al., 2011) la evaluación generada de una demanda externa o de una decisión interna consiste en producir conocimientos sobre la acción pasada o sobre el estado actual de las cosas, a fin de orientar la acción futura. En el caso de la investigación, la evaluación no se limita a una cuestión de ciencia sino también de acción y de poder. A su vez, Zarka (2009) asegura que el poder no es ni ha sido indiferente al saber, y ha encontrado en la evaluación un instrumento para asegurarse una dominación universal sobre todos los sectores de las actividades que se inscriben en las instituciones, los organismos, los establecimientos públicos o privados, y sobre todos los órdenes de la sociedad.

Desde una perspectiva diferente a la de Friedberg, Simonet (2009) define a la evaluación como un proceso social elaborado que toma en consideración valores culturales, institucionales y de los individuos, en términos de implicación voluntaria y recíproca. Esta condición, además de permitirle situarse en relación con los sistemas de valores, la habilita a apoyarse en valores y así, poder reproducir o producir valores.

$\mathrm{Si}$ bien todo proyecto de evaluación encierra intenciones de orden político e institucional, y persigue básicamente la realización de un conjunto indeterminado de valores y 
resultados, los alcances de la evaluación sólo pueden ser pensados en términos de estrategias orientadas al logro de metas provisionales y poco determinadas que puedan cumplirse en una duración-tiempo y en un futuro difícilmente programable con precisión. En opinión de Maury et. al. (2011), lo que las evaluaciones tienen en común no es precisamente la búsqueda de la verdad ni de la pertinencia de decisiones; lo que verdaderamente interesa son los procesos estratégicos que permitan aumentar los márgenes de maniobra de los involucrados. Para los autores se trata de un proceso negociado en el marco de las fuerzas de poder. Sin limitarla a ser sólo una práctica estratégica, la evaluación también es una práctica cultural ya que produce conocimientos, representaciones y valores.

El intento por evaluar situaciones, prácticas y comportamientos individuales y colectivos, con la expectativa de obtener resultados socialmente útiles, dependerá de la relevancia y del conocimiento que se tenga de la particularidad de los sistemas culturales de referencia presentes en el proceso que se pretende evaluar. Al contar con un dispositivo de esta naturaleza, la evaluación puede incluso hacer uso -de manera crítica y bien delimitada- de aquellos procedimientos técnicos que se consideren pertinentes -comprendidos aquéllos de los que se vale la institución-, para el tipo de análisis valorativo que se pretenda emprender.

La atención que se preste a los sistemas de referencia presentes en la evaluación -particularmente los relacionados con la complejidad que encierra el campo de las ciencias sociales, la complejidad de su proceso de institucionalización, así como la naturaleza social de las demandas de la evaluación- puede garantizar la pertinencia y fecundidad de las estrategias metodológicas de valoración de todo tipo de fenómenos asociados a la investigación de lo social. En esta perspectiva, la evaluación se constituye en una plataforma de desarrollo innovador en este y en otros campos de conocimiento.

La particularidad que encierra cada uno de los sistemas de referencia de la evaluación de la investigación en ciencias sociales abre la posibilidad de definir con mayor precisión tanto los objetivos de esta última, como también la trayectoria metodológica y conceptual que resulte más pertinente y adecuada para evaluarla. A continuación se expone un acercamiento a los sistemas de referencia en el caso de la investigación en ciencias sociales.

- El campo científico-intelectual. Las distintas formas de apropiación del conocimiento de lo social se configuran en un complejo sistema de relaciones, de predominio y competencia. Una situación que invita a plantearse controversias tales como: el qué se investiga, y si tal selección atiende a la diversidad de debates epistemológicos, teóricos y metodológicos existentes sobre las distintas formas de cómo ha sido y es interpretada una determinada problemática social; y además, si se concibe al quehacer cotidiano de la investigación social como una actividad lineal e inmutable, o bien diferenciable en la medida que está sujeta a distintos contextos, modelos y vínculos sociales sobre los cuales se ha desarrollado históricamente.

- El entorno institucional como un conjunto de sub-sistemas predeterminados de posiciones, a las que sólo es posible acceder con el cumplimiento de dos requisitos. Primero, la distinción de clases de agentes provistos de cualidades determinadas (socialmente constituidas), tal como si fuera una estructura de mercado de trabajo respaldada por una relación de oferta-demanda. Segundo, un funcionamiento ordenado sobre la base de puestos, funciones y jerarquías. Cada sub-sistema se sostiene gracias a la presencia de tres componentes: a) la posición que guardan los investigadores frente a la estratificación 
social de su quehacer institucional y, por ende, de su vínculo con los sectores de poder; b) las relaciones objetivas de competencia que establecen los grupos de académicos por la obtención de la legitimidad intelectual, y c) el conjunto de intereses con respecto a la producción de conocimiento, y puestos en juego por el investigador.

- Sobre la naturaleza institucional de la evaluación, su acción y alcance restringidos al control y al mantenimiento del orden establecido. La importancia de esclarecer la función de los grupos que la llevan a cabo, así como su preocupación más por cuestiones de procedimiento que sobre los rasgos propiamente científico-sociales puestos en juego en la investigación. La distinción y explicitación sobre el sentido atribuido al quehacer científico y al desempeño de los actores encargados de promover esta actividad. Por último, quiénes son estos actores en términos de grupos de interés que comparten y compiten por una posición en un espacio constituido por valores predeterminados, legitimando además representaciones a-históricas sobre la actividad de investigación.

—La evaluación en el campo de la investigación en ciencias sociales, más que establecer recomendaciones de tipo normativo que respalden decisiones institucionales y de política, debiera formularse en términos de una propuesta que ponga de relieve las tensiones, contradicciones y ambigüedades -así como sus posibles transformaciones- que en conjunto subyacen en la producción y en los procesos tanto de la vida institucional, como de los sistemas sociales involucrados en el mundo académico y científico. En su dimensión institucional, "El problema de la evaluación debe situarse en campos educativos [y académicos] que incluyen relaciones de poder que, en sus orígenes, contribuyen a los procesos de producción social y reproducción, así como a la creación de capacidades humanas" (Popkewitz, 1992, p. 97).

En su dimensión política, la evaluación de la investigación universitaria en ciencias sociales debiera definirse como una "epistemología social de la investigación" cuya función fuera la de:

[...] averiguar cómo el gobierno del Estado y el autogobierno de los individuos están entrela-zados en la producción de conocimiento. [...] Las pretensiones de veracidad en la evaluación no son sólo los criterios lógicos de interpretación y de coherencia, sino también normas sobre la verdad construidas socialmente. Las normas tienen el poder de construir identidades y capa-cidades sociales. (Popkewitz, 1992, p. 102)

En este sentido, el alcance de la evaluación sobre el futuro se da en la medida que se aclaren precisamente los límites y contingencias del pasado. Sólo desde la perspectiva del razonamiento, el carácter histórico y de coyuntura de la investigación social podrán convertirse en una herramienta para elegir, a partir de la negación de todo aquello que en el pasado haya sido identificado como límite o dilema (Popkewitz, 1992, pp. 108-110).

La valoración del trabajo y de los productos científicos derivados de la investigación social requiere, no obstante, de un régimen relativamente institucionalizado, y de un tipo de evaluación que permita el establecimiento de instancias de observación, juicio y producción de señales para el desarrollo de un sistema investigación cuya función principal sea investigar para ampliar el conocimiento sobre lo social y su movimiento, más no sólo para "resolver problemas" o exigencias del sector público y de la coyuntura. 
En este sentido, el punto de partida de la evaluación es constituirse como un discurso sobre la investigación que examine los modos en que esta actividad atestigua o bien oculta las condiciones sociales, institucionales, culturales y científicas bajo las cuales se desarrolla; una evaluación que lejos de medir inercias, permita conocer las potencialidades transformadoras de la investigación social.

\section{Reflexión final}

Tal como nos lo hemos planteado a lo largo de este trabajo, pensar en abrir posibilidades para la evaluación de la investigación social nos enfrenta a la ineludible tarea de reformular los puntos de partida que hasta la fecha han respaldado tanto a la investigación como a la evaluación, ambas como actividades sociales con sus respectivos referentes de realidad y de conocimiento.

En esta tarea de abordar la evaluación como proceso, además de desafiar a lo instituido social y culturalmente tanto en el plano de la interacción social como en el campo formal de la ciencia y de las prácticas de investigación, está presente la ineludible condición generada por la intersubjetividad cotidiana, rasgo propio de los individuos, grupos, comunidades y organizaciones. Una subjetividad cuya complejidad forma parte no sólo de la realidad de cada una de estas entidades, sino que es intrínseca a toda forma de vida social. Encarar la inercia institucional no representa mayor dificultad que la planteada precisamente por Claudine Haroche, quien habla sobre el "inevaluable presente en todo individuo" (2010, p. 57), es decir, la singularidad adquirida por cada uno gracias a la vivencia personal.

Frente a una evaluación-control sujeta a cifras, modelos, comparaciones, reglas, y a programaciones calculables y medibles que anulan la subjetividad constitutiva de los individuos, sólo puede esperarse el conformismo y el desarrollo de un espíritu competitivo insustancial e intrascendente. La alternativa para la evaluación se plantea más allá de la inmediata resistencia institucional para delinearse más bien -como lo denomina Haroche (2010) - en una "resistencia intelectual" respaldada por la libertad, la creatividad, la innovación y la audacia exigidas por la actividad y la experiencia de pensar. Un comportamiento que requiere de paciencia frente a la falta de resonancia, de fuerza de carácter, determinación y de confianza en sí mismo ante la ausencia de reconocimiento.

Ante la enorme cantidad y la vertiginosa velocidad con las que fluye la información en la sociedad contemporánea actual, gana terreno un clima de incertidumbre e inestabilidad frente al conocimiento; una dinámica que plantea la necesidad de incrementar el compromiso de pensar lo social de manera crítica y reflexiva, más no ya como una práctica formal, conformista y competitiva. En estas circunstancias, la evaluación como proceso se enfrenta a la necesidad de atender las particularidades del pensamiento reflexivo y crítico, así como las nuevas condiciones en las el conocimiento se produce.

\section{Bibliografía}

— Ardoino, J. y Berger, G. (1998). “La evaluación como interpretación” [traducido por la Dirección de Extensión y Difusión Universitarias, con la colaboración de Fernanda I]. México, D. F.: Universidad Iberoamericana. Recuperado de http://www.inspeccionarticuladadigital.com/uploads/ardoino.pdf 
- Birman, J. (2010). "L'éclipse du sujet et de la singularité dans le discours de l'évaluation". Cahiers internationaux de sociologie, 1 (128-129), pp. 217-244. Recuperado de http://www.cairn.info/revue-cahiers-internationaux-desociologie-2010-1-page-217.htm

- Demarteau, M. (2007). "L'évaluation: toute une histoire". La Santé de l'homme, (390), pp. 26-30. Recuperado de http://inpes.santepubliquefrance.fr/SLH/articles/390/04.htm 17 abril 2017

— Early, A. (2016). “Évaluer la recherche?”. En AAVV. L'évaluation de la recherche en question(s) (pp. 57-66). Bruselas: Académie royale des Sciences, des Lettres et des Beaux-Arts de Belgique.

— Giddens, A. (1994). Consecuencias de la modernidad. Madrid: Alianza Editorial.

- Giddens, A. (2000). Modernidad e identidad del yo. El yo y la sociedad en la época contemporánea. Barcelona: Ediciones Península

— Giddens, A. (2001). "Vivir en una sociedad postradicional". En Beck, U., Giddens, A. y Lash, S. (2001). Modernización reflexiva Política, tradición y estética en el orden social moderno. Madrid: Alianza Editorial.

- Habermas, J. (1987). Teoría y praxis. Estudios de Filosofía social. Madrid: Ed. Tecnos.

- Haroche, C. (2010). "L'inévaluable dans une société de défiance". Cahiers internationaux de sociologie, 1 (128-129), pp. 53-78. Recuperado de http://www.cairn. info/revue-cahiers-internationaux-de-sociologie-2010-1-page-53.htm

— Hintze J. (2001). "Control y evaluación de gestión y resultados". DAAPGE. Documentos y Aportes en Administración Pública y Gestión Estatal, (1), pp. 27-57. Recuperado de https://dialnet.unirioja.es/servlet/articulo?codigo=3991033

— Leclercq, B. (2016). "Évolutions récentes de l'évaluation de la recherche. Quelques concepts pertinents". En AAVV. L'évaluation de la recherche en question(s) (pp. 13-22). Bruselas: Académie royale des Sciences, des Lettres et des Beaux-Arts de Belgique.

— Maury, C., Demailly, L., Mosse, Ph., Devinau, J. (2011). “Qu'est-ce que l'évaluation d'un point de vue sociologique? D'abord une pratique stratégique”. Recuperado de http://www.knowandpol.eu/Qu-est-ce-que-l-evaluation-d-un.html

— Popkewitz, Th. (1992). "Algunos problemas y problemáticas en la producción de la evaluación". Revista de Educación, (299), pp. 95-118

— Popkewitz, Th. (1988). Paradigma e ideología en investigación educativa. Madrid: Ed. Mondadori.

— Simonet, F. (2009). "L'évaluation: objet de standardisation des pratiques sociales". Cités, (37), pp. 91-100. Recuperado de http://www.cairn.info/revue-cites-20091-page-91.htm

—Zarka, Y. Ch. (2009). "Éditorial. Qu’est-ce que tyranniser le savoir?”. Cités (37), pp. 3-6. Recuperado de http://www.cairn.info/revue-cites-2009-1-page-3.htm 